\title{
Predictors of blood pressure control in patients with resistant hypertension
}

\author{
Pales J, Kamensky G, Payer J \\ 5th Department of Internal Medicine, Faculty of Medicine of Comenius University and University Hospital Bratislava, \\ Bratislava, Slovakia, University Hospital Bratislava, Hospital Ruzinov, Bratislava, Slovakia. jan.pales@gmail.com
}

\begin{abstract}
BACKGROUND: Uncontrolled resistant hypertension $(\mathrm{RH})$ defined by the mean 24-hour ambulatory blood pressure (ABPM) represents an independent risk factor in hypertensive patients. Predictors of blood pressure (BP) control in $\mathrm{RH}$ are not yet clearly defined.

OBJECTIVES: To evaluate the predictors of BP control in RH patients with repeated ABPM measurements. METHODS: 114 consecutive patients from outpatient cardiology office fulfilling criteria for RH (office BP $\geq 140$ and/or $90 \mathrm{mmHg}$, with treatment of $\geq 3$ antihypertensive drugs, including diuretic, or controlled BP with $>3$ drugs), with two consecutive ABPM studies were compared in clinical characteristics according to BP control assessed by ABPM

RESULTS: After the second ABPM, BP was controlled in $25.4 \%$ of patients; the remaining $74.6 \%$ were classified as uncontrolled. In the uncontrolled BP group, systolic office BP was $140.91 \pm 16.71 \mathrm{mmHg}$, diastolic 81.26 $\pm 10.92 \mathrm{mmHg}$. In ABPM, systolic was $145.11 \pm 13.65 \mathrm{mmHg}$, diastolic $81.26 \pm 10.92 \mathrm{mmHg}$. Compared to the controlled BP group, in the uncontrolled group the age was higher $72.32 \pm 10.89$ years $(p=0.047)$, baseline average real variability of systolic BP was lower $12.66 \pm 3.08$ vs. $14.52 \pm 3.53(p=0.013)$, no significant difference in baseline standard deviation of systolic BP changes was found.

CONCLUSION: Higher office BP, older age, and increased short term BP variability were associated with an uncontrolled hypertension. Stronger association was found with baseline average real variability rather than standard deviation. No significant differences were found in the dipping status and other clinical characteristics (Tab. 6, Fig. 1, Ref. 28). Text in PDF www.elis.sk.

KEY WORDS: resistant hypertension, ambulatory blood pressure monitoring, blood pressure variability.
\end{abstract}

\section{Introduction}

Resistant hypertension (RH) is defined as blood pressure (BP) remaining above the goal despite treatment with at least three antihypertensive agents including diuretic (if tolerated) or controlled blood pressure that requires four or more agents.

Patients with resistant hypertension have been identified to have significantly higher risk of adverse cardiovascular events (death, myocardial infarction, heart failure, stroke, chronic kidney disease) compared to the hypertensive patients without RH $(1,2)$.

Estimations of RH prevalence vary greatly in different studies. Large retrospective studies have shown the prevalence of RH around $12-15 \%$ in general hypertensive population $(3,4,5)$. Other studies have found prevalence of resistant hypertension in treated patients up to $50 \%$ (1). Recent large meta-analysis found the

5th Department of Internal Medicine, Faculty of Medicine of Comenius University and University Hospital Bratislava, Bratislava, Slovakia, University Hospital Bratislava, Hospital Ruzinov, Bratislava, Slovakia

Address for correspondence: J. Pales, MD, 5th Department of Internal Medicine, Faculty of Medicine of Comenius University and University Hospital Bratislava, Bratislava, Slovakia University Hospital Bratislava, Hospital Ruzinov, Ruzinovska 6, SK-826 06 Bratislava, Slovakia. Phone: +421.2.48234108, Fax: +421.2.48234110 prevalence of RH to be 10-20\% in treated hypertensive patients (7). The variability of prevalence can be attributed to patient selection and, more importantly, to the capability to rule out pseudoresistance caused by white-coat hypertension, nonadherence and suboptimal dosing. In a recent pooled analysis, prevalence rate of $10.1 \%$ was found for uncontrolled resistant hypertension among individuals treated for hypertension (8).

Diagnosis of RH is based mostly on office blood pressure measurement. There is, however an increasing evidence suggesting the importance of ambulatory blood pressure monitoring (ABPM), which allows to differentiate true hypertension and masked hypertension and to exclude white-coat hypertension. This is particularly important in patients with resistant hypertension (diagnosed by office BP), as the proportion of white-coat hypertension in these patients was found to be more than $35 \%$ (9). Inability to reach the target AMBP values in RH patients was found to stratify cardiovascular risk better than the office BP measurement (10). Furthermore, in the patients with $\mathrm{RH}$, the mean ambulatory BP was identified as an independent predictor of fatal and nonfatal cardiovascular events (11).

The risk factors of the resistant hypertension and predictors of blood pressure control in the RH patients are not yet clearly defined. Previous studies have shown that true RH is associated with a higher office blood pressure, longer hypertension duration, older 


\section{$571-576$}

age, diabetes, dyslipidemia, reduced renal function, microalbuminuria, abdominal obesity, left-ventricular hypertrophy $(4,12)$. In recent years, diurnal profile of the $\mathrm{RH}$ patients has been studied using ABPM. Non-dipping was found to be more prevalent and a higher difference was found between office and ambulatory blood pressure in RH. The improvement in dipping status was found to correlate with an improved BP control (13). However, the value of short term blood pressure variability in RH patients has not yet been assessed. An increased intraindividual BP variability has been shown in some studies to predict cardiovascular events and mortality independently of the mean BP value $(14,15)$.

The aim of this study was to evaluate the predictors of blood pressure control in RH patients utilizing repeated ABPM measurements.

\section{Patients and methods}

The subjects were selected from the hypertensive patients of the outpatient cardiology office of the University Hospital Bratislava in the period from April 2012 to December 2014. Patients fulfilling RH criteria were identified - office systolic blood pressure (SBP) of more than $140 \mathrm{mmHg}$ or diastolic blood pressure (DBP) of more than $90 \mathrm{mmHg}$ despite the treatment of at least 3 antihypertensive drugs; or patients with BP controlled with 4 or more medications. For further analysis, only patients with a complete clinical information and two subsequent visits with at least two consecutive ABPM measurements of sufficient quality in periods of 6-12 months were selected. All the patients have three previous office BP measurements. The patients with secondary hypertension and non- adherence to treatment were excluded.

\section{Variables}

All analyzed patients were required to have a complete history, physical examination including the office BP measurements and laboratory tests. The recorded variables were - sex, age, body mass index, cardiovascular risk factors (smoking, diabetes, dyslipidemia), target organ damage (microalbuminuria, albumin/ creatinine ratio, left ventricular hypertrophy), cardiovascular disease history (ischemic heart disease, cerebrovascular disease, prior coronary revascularization, peripheral arterial occlusive disease (PAOD). The recorded laboratory values were glucose, potassium, sodium, creatinine, urea, total cholesterol, LDL-cholesterol, HDLcholesterol and triacylglycerols. Antihypertensive treatment was categorized by medication classes. In all the classes, morning and evening drug administration were recorded.

\section{Blood pressure monitoring}

Office BP was measured after 5-minute rest in a sitting position with a calibrated mercury sphygmomanometer or an oscillometric device. The 24-hour ambulatory BP was measured with the calibrated SunTech device in the 30-minute intervals during daytime and in the 60-minute intervals in nighttime (set to 10:00 $\mathrm{PM}$ to $06: 59 \mathrm{AM}$ ). Valid measurements were required to have $>$ $70 \%$ measurements recorded. The patients were instructed to maintain their usual daily activities. Based on the ABPM results (mean 24-hour $\mathrm{BP} \geq 130 / 80 \mathrm{mmHg}$ ), the patients were divided into categories of controlled and uncontrolled hypertension.

The 24 hour BP variability was evaluated by calculating ABPM standard deviation (SD). In order to decrease the influence of long term BP daily fluctuation, the average real variability (ARV) was calculated as the average of the absolute differences between consecutive BP measurements over 24 hour, therefore more properly reflecting measure to measure variability. Furthermore, the dipping status was determined. The patients were characterized according to the nighttime $\mathrm{BP}$ as dippers (BP decline $>10 \%$ ), non-dippers (BP decline between 0 and $10 \%$ ), and reverse dippers (increase in BP during nighttime).

The variability of office BP was quantified by calculating SD and ARV of systolic and diastolic BP measured in two visits with $\mathrm{ABPM}$ and the previous three office BP measurements in past three years in the intervals of $6-8$ months.

\section{Statistical analysis}

Quantitative variables are presented as the mean $\pm \mathrm{SD}$, qualitative variables as percentages. Differences in qualitative variables were compared by non-parametric $\chi^{2}$ test and Wilcoxon signedrank test. Differences in quantitative data were compared by the Student's paired t-test. Quantitative and qualitative variables were compared by an independent t-test. A level of significance was $p$ $\leq 0.05$. For statistical analyses, the SPSS version 21 (SPSS Inc.) was used.

\section{Results}

Totally, 114 consecutive patients with RH were included in the analysis - 56 men and 58 women. The mean age was $71.2 \pm$ 11.0 years, $(68.86 \pm 12.27$ years in men and $73.41 \pm 9.14$ years in women). The mean baseline office BP was $139 \pm 16 / 81 \pm 11$ $\mathrm{mmHg}$, the mean baseline 24-hour ABPM was $142 \pm 14 \mathrm{mmHg}$. Obesity (body mass index $\geq 30 \mathrm{~kg} / \mathrm{m}^{2}$ ) was present in $51.8 \%$ of patients, current smoking in $7.0 \%$ and smoking history in $27.2 \%$. Diabetes mellitus was present in $54 \%$, ischemic heart disease in $64.9 \%$, previous myocardial infarction in $13.2 \%$, left ventricular hypertrophy assessed by echocardiography in $35 \%$, cerebrovascular event in $13.2 \%$, renal insufficiency in $21.9 \%$, peripheral

Tab. 1. Differences in office BP, mean 24-hour BP and blood pressure variability between in the first and the second visit.

\begin{tabular}{lccc}
\hline Variable & $\begin{array}{c}\text { First } \\
\text { measurement }\end{array}$ & $\begin{array}{c}\text { Second } \\
\text { measurement }\end{array}$ & $\mathrm{p}$ \\
\hline Office SBP, mmHg & $138.66 \pm 16.30$ & $137.44 \pm 117.60$ & 0.496 \\
\hline Office DBP, $\mathrm{mmHg}$ & $80.90 \pm 10.78$ & $79.69 \pm 9.96$ & 0.212 \\
\hline${\text { Office HR, } \text { min. }^{-1}}^{\text {24-hour mean SBP, } \mathrm{mmHg}}$ & $68.48 \pm 10.48$ & $68.18 \pm 8.65$ & 0.719 \\
\hline 24-hour mean DBP, $\mathrm{mmHg}$ & $72.19 \pm 14.04$ & $139.290 \pm 15.14$ & 0.048 \\
\hline 24-hour mean HR, min. $^{-1}$ & $67.64 \pm 9.47$ & $70.99 \pm 9.89$ & 0.011 \\
\hline 24-hour SD & $17.80 \pm 4.16$ & $18.28 \pm 6.14$ & 0.852 \\
\hline 24-hour ARV & $14.045 \pm 3.50$ & $14.032 \pm 3.84$ & 0.968 \\
\hline
\end{tabular}

Values are mean $\pm \mathrm{SD}, \mathrm{SBP}$ - systolic blood pressure, $\mathrm{DBP}$ - diastolic blood pressure, $\mathrm{HR}$ - heart rate, $\mathrm{SD}$ - standard deviation, $\mathrm{ARV}$ - average real variability 
Tab. 2. Differences in clinical characteristics in the first visit between patients with controlled and uncontrolled 24-hour systolic blood pressure.

\begin{tabular}{|c|c|c|c|}
\hline Variable & $\begin{array}{c}\text { Controlled BP } \\
n=29(25.4 \%)\end{array}$ & $\begin{array}{c}\text { Uncontrolled BP } \\
\mathrm{n}=85(74.6 \%)\end{array}$ & $\mathrm{p}$ \\
\hline Age, years & $67.82 \pm 10.76$ & $72.32 \pm 10.89$ & 0.047 \\
\hline Sex, male \% & 48.3 & 49.4 & 0.916 \\
\hline $\mathrm{DM}, \%$ & 58.6 & 52.9 & 0.600 \\
\hline Smoking \% & 13.8 & 4.7 & 0.240 \\
\hline Coronary heart disease, $\%$ & 55.2 & 68.2 & 0.206 \\
\hline Creatinine, umol/1 & $90.75 \pm 36.72$ & $95.12 \pm 30.11$ & 0.546 \\
\hline Glucose, $\mathrm{mmol} / \mathrm{l}$ & $6.78 \pm 1.63$ & $7.20 \pm 2.27$ & 0.380 \\
\hline HbA1c & $7.100 \pm 1.63$ & $8.500 \pm 1.32$ & 0.053 \\
\hline Cholesterol, mmol/1 & $4.38 \pm 0.73$ & $4.23 \pm 0.93$ & 0.462 \\
\hline HDL, mmol/l & $1.29 \pm 0.28$ & $1.18 \pm 0.28$ & 0.078 \\
\hline LDL, mmol/l & $2.50 \pm 0.72$ & $2.60 \pm 0.99$ & 0.658 \\
\hline TAG, mmol/l & $1.50 \pm 0.58$ & $1.57 \pm 0.71$ & 0.644 \\
\hline Albumin/creatinine & $3.77 \pm 7.94$ & $6.21 \pm 12.08$ & 0.496 \\
\hline Average number of AH drugs & $4.79 \pm 1.01$ & $5.094 \pm 1.269$ & 0.250 \\
\hline Treatment with $>4$ drugs, $\%$ & 51.9 & 63.5 & 0.265 \\
\hline Average morning drugs & $2.90 \pm 0.86$ & $3.08 \pm 1.11$ & 0.415 \\
\hline Average evening drugs & $2.79 \pm 1.01$ & $2.80 \pm 1.06$ & 0.975 \\
\hline
\end{tabular}

Tab. 3. Differences in clinical characteristics in the second visit between patients with controlled and uncontrolled 24-hour systolic blood pressure.

\begin{tabular}{lccc}
\hline Variable & $\begin{array}{c}\text { Controlled BP } \\
\mathrm{n}=29(25.4 \%)\end{array}$ & $\begin{array}{c}\text { Uncontrolled BP } \\
\mathrm{n}=85(74.6 \%)\end{array}$ & $\mathrm{p}$ \\
\hline Creatinine, umol/l & $90.21 \pm 31.17$ & $96.23 \pm 25.61$ & 0.311 \\
\hline Glucose, $\mathrm{mmol} / \mathrm{l}$ & $6.79 \pm 1.63$ & $7.55 \pm 3.12$ & 0.105 \\
\hline HbA1c & $6.77 \pm 0.46$ & $7.92 \pm 1.92$ & 0.356 \\
\hline Cholesterol, mmol/1 & $4.12 \pm 0.62$ & $4.16 \pm 0.97$ & 0.821 \\
\hline $\mathrm{HDL}, \mathrm{mmol} / \mathrm{l}$ & $1.23 \pm 0.25$ & $1.19 \pm 0.37$ & 0.655 \\
\hline LDL, mmol/l & $2.75 \pm 0.51$ & $2.65 \pm 0.89$ & 0.615 \\
\hline TAG, mmol/l & $1.37 \pm 0.74$ & $1.66 \pm 0.88$ & 0.108 \\
\hline Albumin/creatinine & $6.77 \pm 0.46$ & $7.92 \pm 1.92$ & 0.020 \\
\hline AveragenumberofAHdrugs & $4.86 \pm 1.03$ & $5.24 \pm 1.22$ & 0.143 \\
\hline Treatment with $>4$ drugs, $\%$ & 55.2 & 74.1 & 0.056 \\
\hline Average morning drugs & $2.72 \pm 0.96$ & $3.27 \pm 1.14$ & 0.022 \\
\hline Average evening drugs & $3.00 \pm 0.96$ & $3.00 \pm 1.06$ & 1.000 \\
\hline
\end{tabular}

Values are mean $\pm \mathrm{SD}, \mathrm{BP}-$ blood pressure, $\mathrm{HDL}$ - high-density lipoprotein, $\mathrm{LDL}-$ low-density lipoprotein, TAG - triacylglycerol, HbAlc - hemoglobin Alc

arterial occlusive disease in $6.1 \%$. Men were significantly more likely to smoke $(p=0.002)$, and to have PAOD $(p=0.006)$, the higher prevalence of renal insufficiency and target organ damage in men was non-significant.

In the first office BP measurement, $39.5 \%$ of the patients had $\mathrm{BP}$ controlled (BP $\leq 140$ or $90 \mathrm{mmHg}$ ). These patients however required 4 or more antihypertensive medications and were classified as RH. The remaining $60.5 \%$ of the patients were considered as having uncontrolled BP. In the first ABPM, there were 86.6 $\%$ of the patients with the mean 24-hour BP of $\geq 130$ and/or 80 $\mathrm{mmHg}$ (Fig. 1). Controlled office BP with uncontrolled ABPM was recorded in $31.6 \%$ of the patients. Uncontrolled office BP with controlled ABPM was recorded in $5.3 \%$ of the patients. The patients in this subgroup were not found to differ significantly in any of the risk factors, and treatment characteristics.

In the subsequent office BP measurement, $43.0 \%$ of the patients had a controlled BP. In the ABPM $25.4 \%$ of the patients were controlled and $74.6 \%$ uncontrolled (Fig. 1). Controlled office BP with uncontrolled ABPM was recorded in $27.2 \%$ of the patients. Prevalence of uncontrolled office BP with controlled ABPM was $9.6 \%$. Compared to the first visit, office SBP and DBP were lowered non-significantly. However, the ABMP mean 24-hour SBP and DBP decreased significantly ( $\mathrm{p}=0.048$ and 0.011 , respectively). No significant change between visits was recorded in BP variability indices (Tab. 1). In the laboratory results, only non-significant overall improvement in blood lipids and albumin-creatinine ratio were recorded (Tabs 2 and 3 ).

The median number of antihypertensive medication classes was 4 in both visits. In the first visit, the patients were treated with diuretics (by definition), ACE inhibitors (98\%), beta blockers $(84.2 \%)$, calcium channel blockers ( $72 \%)$, mineralocorticoid receptor antagonists $(19 \%)$ and other unclassified centrally and peripherally active antihypertensives ( $81 \%)$.

In the second visit, more patients were treated with more than 4 antihypertensive medications -69.2 vs $60.5 \%(p=0.03)$, the average amount of medications had not increased significantly (5.02 vs 5.14). More medications were administered in the evening, 3.00 vs $2.80(p=0.005)$. No significant change was recorded in the morning medications (3.04 vs 3.13). Regarding medication classes, more centrally and peripherally active antihypertensives were used 91 vs $81 \%(p=0.001)$. Mineralocorticoid receptor antagonists increased non-significantly (24 vs $19 \%$ ). No significant change was found in the other medication classes.

After the second ABPM, the patients were classified into the groups according to the mean SBP and DBP results. There were 29 patients $(25.4 \%)$ with controlled BP, 85 patients $(74.6 \%)$ with uncontrolled BP, 22 patients (19.3\%) with improved BP control, and 8 patients $(7.0 \%)$ with worsened BP control from the first visit.

Uncontrolled hypertension was associated with older age (72.3 vs 67.8 years) and higher office SBP in both visits. Other clinical characteristics including cardiovascular risk factors, cardiovascular disease history and target organ damage were not significantly different (Tab. 2). The average number of antihypertensives was generally higher in the uncontrolled BP group, particularly in the morning treatment (Tabs 2 and 3). No significant difference was found in the antihypertensive medication classes. However, the increase in centrally and peripherally active antihypertensives was found in the uncontrolled BP group $(p=0.03)$, whereas in the controlled BP, there was no significant difference.

The circadian pattern showed no significant difference in dipping status between the controlled and uncontrolled BP groups, there were no significant changes between the visits. Only an insignificant trend towards an increase of non-dipping in the uncontrolled BP group was found (Tabs 4 and 5).

Regarding the ABPM short term variability comparison, in the first visit, ARV tend to be lower in the controlled BP group 
Tab. 4. Differences in office BP, mean 24-hour BP and blood pressure variability in the first visit between patients with controlled and uncontrolled 24-hour systolic blood pressure.

\begin{tabular}{lccc}
\hline Variable & $\begin{array}{c}\text { Controlled BP } \\
\mathrm{n}=29(25.4 \%)\end{array}$ & $\begin{array}{c}\text { Uncontrolled BP } \\
\mathrm{n}=85(74.6 \%)\end{array}$ & $\mathrm{p}$ \\
\hline Office SBP, $\mathrm{mmHg}$ & $132.07 \pm 13.20$ & $140.91 \pm 16.71$ & 0.011 \\
\hline Office DBP, $\mathrm{mmHg}$ & $79.83 \pm 10.48$ & $81.26 \pm 10.92$ & 0.539 \\
\hline Office HR, min. $^{-1}$ & $68.90 \pm 11.84$ & $68.34 \pm 10.04$ & 0.807 \\
\hline 24-hour mean SBP, $\mathrm{mmHg}$ & $133.66 \pm 11.64$ & $145.11 \pm 13.65$ & $<0.001$ \\
\hline 24-hour mean DBP, mmHg & $73.21 \pm 11.41$ & $72.81 \pm 10.23$ & 0.862 \\
\hline 24-hour mean HR, min. ${ }^{-1}$ & $71.48 \pm 11.28$ & $66.33 \pm 8.45$ & 0.011 \\
\hline 24-hour SD & $17.04 .00 \pm 17.04$ & $18.06 \pm 4.43$ & 0.257 \\
\hline 24-hour ARV & $12.66 \pm 3.08$ & $14.52 \pm 3.53$ & 0.013 \\
\hline Diastolic dipping status & 24.1 & 25.9 & 0.852 \\
\hline Dippers DBP, \% & 31.0 & 31.8 & 0.816 \\
\hline Non-dippers DBP, \% & 44.8 & 42.2 & 0.942 \\
\hline $\begin{array}{l}\text { Reverse dippers DBP, } \% \\
\text { Systolic dipping status }\end{array}$ & 20.7 & 27.1 & 0.496 \\
\hline Dippers SBP, \% & 31.0 & 32.9 & 0.850 \\
\hline Non-dippers SBP, \% & 48.3 & 40.0 & 0.436 \\
\hline Reverse dippers SBP, $\%$ & &
\end{tabular}

Values are mean $\pm \mathrm{SD}, \mathrm{BP}$ - blood pressure, $\mathrm{SBP}$ - systolic blood pressure, DBP diastolic blood pressure, HR - heart rate, SD - standard deviation, ARV - average real variability

Tab. 5. Differences in office BP, mean 24-hour BP and blood pressure variability in the second visit between patients with controlled and uncontrolled 24-hour systolic blood pressure.

\begin{tabular}{lccc}
\hline Variable & $\begin{array}{c}\text { Controlled BP } \\
\mathrm{n}=29(25.4 \%)\end{array}$ & $\begin{array}{c}\text { Uncontrolled BP } \\
\mathrm{n}=85(74.6 \%)\end{array}$ & $\mathrm{p}$ \\
\hline Office SBP, $\mathrm{mmHg}$ & $128.48 \pm 15.48$ & $140.49 \pm 17.31$ & 0.001 \\
\hline Office DBP, mmHg & $79.14 \pm 9.07$ & $79.88 \pm 10.29$ & 0.730 \\
\hline Office HR, min. $^{-1}$ & $69.00 \pm 8.69$ & $67.89 \pm 8.67$ & 0.555 \\
\hline 24-hour mean SBP, mmHg & $122.21 \pm 6.04$ & $145.12 \pm 12.70$ & $<0.0001$ \\
\hline 24-hour mean DBP, mmHg & $66.62 \pm 7.76$ & $72.48 \pm 10.13$ & 0.005 \\
\hline 24-hour mean HR, min. ${ }^{-1}$ & $69.59 \pm 8.67$ & $66.76 \pm 8.60$ & 0.131 \\
\hline 24-hour SD & $15.94 \pm 3.84$ & $19.08 \pm 6.57$ & 0.017 \\
\hline 24-hour ARV & $12.61 \pm 3.19$ & $14.51 \pm 3.94$ & 0.021 \\
\hline Diastolic dipping status & 24.1 & 24.7 & 0.951 \\
\hline Dippers, \% & 31.0 & 34.1 & 0.761 \\
\hline Non-dippers, \% & 44.8 & 41.2 & 0.731 \\
\hline $\begin{array}{l}\text { Reverse dippers, \% } \\
\text { Systolic dipping status }\end{array}$ & 27.6 & 23.5 & 0.661 \\
\hline Dippers, \% & 31.0 & 32.9 & 0.850 \\
\hline Non-dippers, \% & 41.4 & 43.5 & 0.840 \\
\hline Reverse dippers, \% & &
\end{tabular}

Values are mean $\pm \mathrm{SD}, \mathrm{BP}$ - blood pressure, $\mathrm{SBP}$ - systolic blood pressure, DBP diastolic blood pressure, $\mathrm{HR}$ - heart rate, $\mathrm{SD}$ - standard deviation, $\mathrm{ARV}$ - average real variability

$(p=0.013)$, whereas SD showed no significant difference (Tab. 4). In the second visit in the controlled BP group, ARV and SD tend to be lower (Tab. 5). The variability of office BP showed no significant difference between the groups (Tab. 6).

\section{Discussion}

The present analysis from the registry of the outpatient cardiology office showed the significance of ambulatory 24-hour blood
Tab. 6. Differences in office BP variability between patients with controlled and uncontrolled 24-hour systolic blood pressure.

\begin{tabular}{lccc}
\hline Variable & $\begin{array}{c}\text { Controlled BP } \\
\mathrm{n}=29(25.4 \%)\end{array}$ & $\begin{array}{c}\text { Uncontrolled BP } \\
\mathrm{n}=85(74.6 \%)\end{array}$ & $\mathrm{p}$ \\
\hline Office SBP SD & $11.89 \pm 5.54$ & $13.11 \pm 7.21$ & 0.410 \\
\hline Office DBP SD & $6.86 \pm 4.24$ & $7.72 \pm 4.17$ & 0.314 \\
\hline Office HR SD & $6.86 \pm 4.24$ & $5.13 \pm 4.18$ & 0.089 \\
\hline Office SBP ARV & $14.47 \pm 8.26$ & $15.08 \pm 8.83$ & 0.745 \\
\hline Office DBP ARV & $7.83 \pm 5.73$ & $8.92 \pm 5.25$ & 0.345 \\
\hline Office HR ARV & $7.21 \pm 4.80$ & $5.15 \pm 4.16$ & 0.029 \\
\hline
\end{tabular}

Values are mean $\pm \mathrm{SD}, \mathrm{SBP}$ - systolic blood pressure, $\mathrm{DBP}$ - diastolic blood pressure, $\mathrm{SD}$ - standard deviation, ARV - average real variability, $\mathrm{HR}$ - heart rate

pressure measurement in the management of high risk patients with resistant hypertension. Moreover, an increased 24-hour BP variability was shown to be associated with a worsened BP control.

Contribution of ABPM to diagnosis and management of $\mathrm{RH}$ is evident from the differences in the uncontrolled office BP and $\operatorname{ABPM}(60.5 \%$ vs $86.6 \%$ in the first visit) (Fig. 1). In the second ABPM, the prevalence of the uncontrolled BP was $74.6 \%$. In the large population study, the uncontrolled BP prevalence of $62 \%$ was found in the RH patients. Uncontrolled hypertension represents a cardiovascular risk factor and needs to be managed.

Moreover, ABPM was shown to be more sensitive in capturing visit to visit $\mathrm{BP}$ variability. Differences in $\mathrm{BP}$ control between the visits showed a significant difference in $\mathrm{ABMP}$, but not in the office BP (Tab. 1). ABPM needs to exclude false RH that is associated with better prognosis (10). In the present study, falsely uncontrolled RH (uncontrolled office BP and controlled ABPM) was found in only $5.3 \%$ of patients ( $9.6 \%$ in the second visit). These patients were all taking 4 or more medications, therefore classified as RH. Also, they did not differ significantly from the rest of the patients. Previous studies with larger cohorts of RH patients showed false $\mathrm{RH}$ to be associated with better $\mathrm{CV}$ risk profile and circadian BP pattern. The analysis in the present study was however limited by the small number of patients.

Dipping status is the most widely used measure to describe $\mathrm{BP}$ variability. In the previous studies, non-dipping was found to be more common in the $\mathrm{RH}$ patients. In the $\mathrm{RH}$ patients, recovery of pathological diurnal rhythm was associated with an improved BP control (13). Moreover, non-dipping is known to be associated with the target organ damage (17), cardiovascular events and mortality (18). Nighttime BP changes were shown to have a higher prognostic value compared to the average 24 hour BP (19). In the present study, no significant association between dipping status and BP control was found. The reason for this might be very high baseline prevalence of non-dipping and reverse dipping of more than $74 \%$, suggesting the possibility of "fixation" of pathological dipping patterns in this high risk patient group. The other possibility might be imprecision in the analysis caused by rigid rather than individually defined nighttime.

Considering the high prevalence of non-dippers, there is notably a high proportion $(98.2 \%)$ of patients that received some of their medications in the evening. Bedtime dosing of antihypertensive medication was associated with a reduced nocturnal BP by several studies (20). Currently, the effect of chronotherapy on 


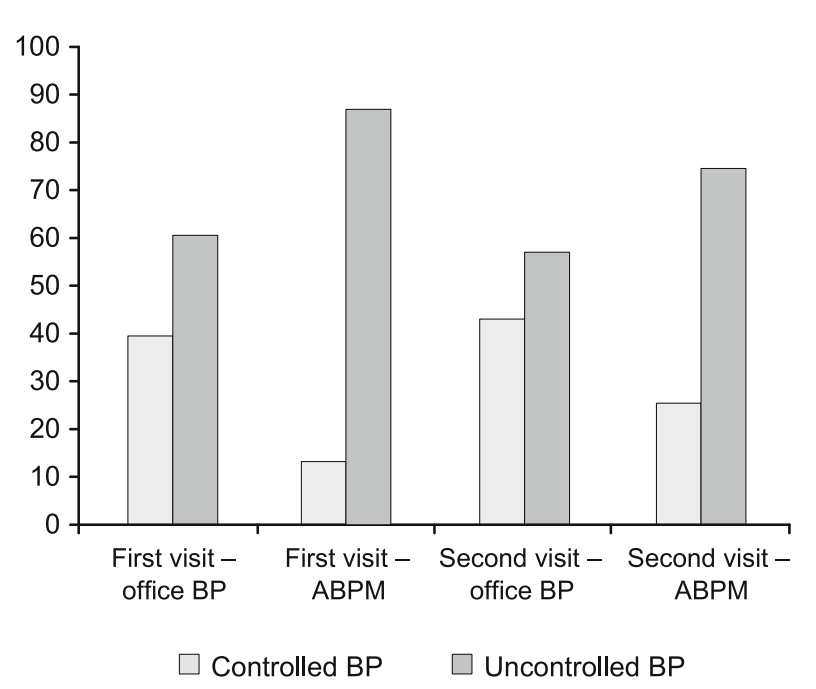

Fig. 1. Differences in controlled and uncontrolled BP prevalence between office BP and 24-hour ABPM. Values are in \% of patients, BP - blood pressure, ABPM - Ambulatory blood pressure monitoring

cardiovascular risk is still unclear (21). In the present study, no significant association was found between the BP control and the treatment timing and intensity.

Short term BP variability is most commonly quantified as the standard deviation. However, by definition, this index shows the dispersion of the BP around the mean and is therefore influenced by the mean BP levels. Although this can be addressed by calculating the coefficient of variation (22) it would still be dependent on underlying $\mathrm{BP}$ trends during the day, such as day-night changes. Short term measure to measure BP variations could be therefore undetected by SD.

The advantage of ARV in assessing BP variability can be attributed to its ability to decrease influence of long term BP fluctuation, thus better reflecting the short term changes in BP. There are several studies available showing better prognostic value of $\mathrm{ARV}$ in CV risk assessment compared to SD and SBP $(16,23)$.

In the present study, short term ARV compared to SD in the first visit was more strongly associated with future BP control in the second visit. ABPM data from the first visit showed lower ARV in the controlled BP group, whereas there was only a weak association with $\mathrm{SD}$. In the second visit, both SD and ARV were associated with the $\mathrm{BP}$ control. There was however no association found between the BP control and the variability of the office BP measurements (Tab. 6).

Blood pressure variability is known to be influenced by sympathetic factors (24). In normotensive patients, higher sympathetic activity was associated with a higher daytime blood pressure variability. In recent studies, short term BP variability quantified as $\mathrm{ARV}$ in patients with treatment-resistant hypertension was shown to decrease significantly after renal sympathetic denervation $(25$, 26). Moreover, higher sympathetic activity was found in refractory hypertension patients (27). Based on these finding, we can hypothesize that sympathetic over activity could be one of the pathogenic factors of uncontrolled RH and ARV. No correlation with the heart rate variability was found in the previous studies. However, some other unknown regularity might be present in variability patterns.
Apart from ABPM parameters, other clinical characteristics did not show a significant difference between the patients with controlled and uncontrolled hypertension. Weak association was found between the uncontrolled BP and higher age, higher $\mathrm{HbA} 1 \mathrm{c}$ and lower HDL. An increased prevalence of treatment with 4 or more drugs was non-significant (Tab. 3).

Recent studies with large cohorts, however showed clear differences between controlled and uncontrolled BP. Uncontrolled $\mathrm{BP}$ was associated with a worse $\mathrm{CV}$ risk profile, more prevalent smoking, diabetes and target organ damage $(4,28)$. The reason for the observed lack of differences in clinical characteristics could be the selection of patients with sufficient amount of clinical data and BP measurements, therefore selecting a group with a rather homogenously high risk CV profile. Moreover, in the past studies, the differences were compared between patients with true $\mathrm{RH}$ and patients with controlled BP, while majority of the patients were treated with 3 medications. The patients with ABPM below the cutoff were therefore mostly classified as white coat hypertension. In the present study, only $4.7 \%$ of the patients were treated with less than 4 medications, all these patients were classified as uncontrolled BP by ABPM.

\section{Conclusion}

Uncontrolled hypertension defined by the mean 24-h ambulatory BP in RH patients, or "true RH" was repeatedly associated with a worsened cardiovascular prognosis. The prevalence and risk factors of uncontrolled resistant hypertension were assessed by several studies. These were predominantly retrospective analyses of large population studies or registries, limited by unaddressed possibility of nonadherence and frequent inadequate not fully up-titrated medical treatment. This resulted in high prevalence of apparent "white coat" RH, therefore comparing uncontrolled RH with mostly substantially lower risk patients. In the present study, all the patients received adequate antihypertensive treatment. The vast majority of patients received 4 or more medications and all of them could be classified as RH after the first ABPM. The observed lack of significant differences in clinical characteristics could be therefore caused by more similar risk profiles of the patients. However, statistical power was also limited due to retrospective study design and small size of patient groups.

The study results reaffirm the pivotal role of ABPM in diagnosing and management of RH patients. The observed laboratory and clinical characteristics have shown only marginal nonsignificant association with blood pressure control. The results suggested that the assessment of short term blood pressure variability in high risk patients with resistant hypertension can predict future blood pressure control, and therefore was able to provide prognostic information. Despite relatively easy derivation of both SD and ARV, w ider clinical application will nevertheless depend on automatization of the calculation. The present generation ABPM recording systems are mostly able to derive the mean BP and SD separately for day and nighttime. Automatic ARV calculation would be another desirable feature in future ABPM monitors. 
571-576

\section{References}

1. Daugherty SL, Powers JD, Magid DJ, Tavel HM, Masoudi FA, Margolis KL et al. Incidence and prognosis of resistant hypertension in hypertensive patients. Circulation 2012; 125 (13): 1635-1642.

2. Smith SM, Gong Y, Handberg E, Messerli FH, Bakris GL, Ahmed A et al. Predictors and outcomes of resistant hypertension among patients with coronary artery disease and hypertension. J Hypertens 2014; 32 (3): 635-643.

3. Persell SD. Prevalence of resistant hypertension in the United States, 2003-2008. Hypertension 2011; 57 (6): 1076-1080.

4. De la Sierra A, Segura J, Banegas JR, Gorostidi M, Juan J, Armario $\mathbf{P}$ et al. Clinical features of 8295 patients with resistant hypertension classified on the basis of ambulatory blood pressure monitoring. Hypertension 2011; 57 (5): 898-902.

5. Sim JJ, Bhandari SK, Shi J, Liu ILA, Calhoun DA, McGlynn EA et al. Characteristics of resistant hypertension in a large, ethnically diverse hypertension population of an integrated health system. Elsevier; 2013, p. 1099-1107.

6. Gupta AK, Nasothimiou EG, Chang CL, Sever PS, Dahlöf B, Poulter NR et al. Baseline predictors of resistant hypertension in the AngloScandinavian Cardiac Outcome Trial (ASCOT): a risk score to identify those at high-risk. J Hypertens 2011; 29 (10): 2004-2013.

7. Achelrod D, Wenzel U, Frey S. Systematic review and meta-analysis of the prevalence of resistant hypertension in treated hypertensive populations. Amer J Hypertens 2014; hpu151.

8. Judd E, Calhoun D. Apparent and true resistant hypertension: definition, prevalence and outcomes. J Hum Hypertens 2014; 28 (8): 463.

9. De la Sierra A. Profile of ambulatory blood pressure in resistant hypertension. Hypertens Res 2013; 36 (7): 565-569.

10. Pierdomenico SD, Lapenna D, Bucci A, Di Tommaso R, Di Mascio R, Manente BM et al. Cardiovascular outcome in treated hypertensive patients with responder, masked, false resistant, and true resistant hypertension. Amer J Hypertens 2005; 18 (11): 1422-1428.

11. Salles GF, Cardoso CR, Muxfeldt ES. Prognostic influence of office and ambulatory blood pressures in resistant hypertension. Arch Intern Med 2008; 168 (21): 2340-2346.

12. Calhoun DA, Jones D, Textor S, Goff DC, Murphy TP, Toto RD et al. Resistant hypertension: diagnosis, evaluation, and treatment a scientific statement from the American Heart Association Professional Education Committee of the Council for High Blood Pressure Research. Hypertension 2008; 51 (6): 1403-1419.

13. Dlesk A, Kamensky G, Stefanik M, Kuzma M, Pernicky M. Treatment efficiency of resistant hypertension in cardiologist's office. Bratisl Lek Listy 2013; 115 (1): 25-29.

14. Hansen TW, Thijs L, Li Y, Boggia J, Kikuya M, Björklund-Bodegård K et al. Prognostic value of reading-to-reading blood pressure variability over 24 hours in 8938 subjects from 11 populations. Hypertension 2010; 55 (4): 1049-1057.

15. Mancia G, Bombelli M, Facchetti R, Madotto F, Corrao G, Trevano FQ et al. Long-term prognostic value of blood pressure variability in the general population results of the Pressioni Arteriose Monitorate e Loro Associazioni Study. Hypertension 2007; 49 (6): 1265-1270.
16. Mena L, Pintos S, Queipo NV, Aizpurua JA, Maestre G, Sulbaran T. A reliable index for the prognostic significance of blood pressure variability. J Hypertens 2005; 23 (3): 505-511.

17. Kawai T, Ohishi M, Kamide K, Nakama C, Onishi M, Ito N et al. Differences between daytime and nighttime blood pressure variability regarding systemic atherosclerotic change and renal function. Hypertens Res 2013; 36 (3): 232-239.

18. Hansen TW, Li Y, Boggia J, Thijs L, Richart T, Staessen JA. Predictive role of the nighttime blood pressure. Hypertension 2011; 57 (1): 3-10.

19. Salles GF, Reboldi G, Fagard RH, Cardoso CRL, Pierdomenico SD, Verdecchia Pet al. Prognostic Effect of the Nocturnal Blood Pressure Fall in Hypertensive Patients The Ambulatory Blood Pressure Collaboration in Patients With Hypertension (ABC-H) Meta-Analysis. Hypertension 2016; 67 (4): 693-700.

20. Hermida RC, Ayala DE, Calvo C, López JE, Mojón A, Fontao MJ et al. Effects of time of day of treatment on ambulatory blood pressure pattern of patients with resistant hypertension. Hypertension 2005; 46 (4): 1053-1059.

21. Schillaci G, Battista F, Settimi L, Schillaci L, Pucci G. Antihypertensive drug treatment and circadian blood pressure rhythm: a review of the role of chronotherapy in hypertension. Curr Pharmaceut Design 2015; 21 (6): 756-772.

22. Parati G, Ochoa JE, Lombardi C, Bilo G. Blood pressure variability: assessment, predictive value, and potential as a therapeutic target. Curr Hypertens Rep 2015; 17 (4): 1-18.

23. Hsu PF, Cheng HM, Wu CH, Sung SH, Chuang SY, Lakatta EG et al. High Short-Term Blood Pressure Variability Predicts Long-Term Cardiovascular Mortality in Untreated Hypertensives But Not in Normotensives. Am J Hypertens 2016; 29 (9): 1-8.

24. Narkiewicz K, Winnicki M, Schroeder K, Phillips BG, Kato M, Cwalina E et al. Relationship Between Muscle Sympathetic Nerve Activity and Diurnal Blood Pressure Profile. Hypertension 2002; 39 (1): 168-172.

25. Miroslawska A, Solbu M, Skjølsvik E, Toft I, Steigen TK. Renal sympathetic denervation: effect on ambulatory blood pressure and blood pressure variability in patients with treatment-resistant hypertension. The ReShape CV-risk study. J Hum Hypertens 2016; 30 (3): 153-157.

26. Ewen S, Dörr O, Ukena C, Linz D, Cremers B, Laufs U et al. Blood pressure variability after catheter-based renal sympathetic denervation in patients with resistant hypertension: J Hypertens 2015; 33 (12): 2512-2518.

27. Dudenbostel T, Acelajado MC, Pisoni R, Li P, Oparil S, Calhoun DA. Refractory Hypertension Evidence of Heightened Sympathetic Activity as a Cause of Antihypertensive Treatment Failure. Hypertension 2015; 66 (1): 126-133.

28. Mancia G, Banegas JR, Segura J, Sobrino J, Rodriguez-Artalejo F, de la Sierra A et al. Effectiveness of blood pressure control outside the medical setting. Commentary. Hypertension 2007; 49 (1).

Received April 18, 2016. Accepted June 1, 2016. 